\title{
Side Panel Crack Fault Diagnosis Research of Large-scale Vibrating Screen Based on Wavelet Analysis
}

\author{
ZHU Qing hui ${ }^{1, a}$, WANG Han ${ }^{2, b}$ \\ ${ }^{1}$ School of Electronic and Electric Engineering,Nanyang Institute of Science and Technology,China \\ ${ }^{2}$ School of Electric Engineering and Information Technology, Damm Schardt Industrial University, \\ Germany \\ a0zhu@163.com, bnfind@126.com
}

Keywords: Fault diagnosis; vibrating screen; wavelet analysis; neural network

\begin{abstract}
The paper takes side panel cracks of large-scale vibrating screen as research object. The side panel vibration signals have been de-noised and the wavelet packet energy feature has been extracted in Matlab with wavelet analysis theory. Meanwhile, the fault characteristics is sent to genetic neural network for identification. The practical manufacturing application shows that this kind of side panel crack fault diagnosis system of large-scale vibrating screen based on wavelet analysis has high fault recognition ability, classification precision and speed.
\end{abstract}

\section{Introduction}

Large-scale line vibrating screen is one of the most common used vibrating screen whose side panel crack fault is most common and the harm brought is maximum ${ }^{[1,2]}$. So, the research and monitoring for side panel crack of large-scale line vibrating screen has very important practical significance. This paper takes the side panel cracks of large-scale line vibrating screen DZK2466 as research object and the fault diagnosis system is designed and constructed which can detect side panel cracks and alarm early. The control system uses the software such as Data Base, Visual Basic and Matlab to process, analyze and identify fault data based on wavelet analysis ${ }^{[3]}$. and neural network algorithm. The system has obtained high fault accuracy diagnosis by practical production use.

\section{System Construction}

The system hardware is constructed mainly with sensors, charge amplifier, microcontroller, upper computer and LCD etc.. The signal is collected by three-axis accelerometer and sonar sensor simultaneously. The charge amplifier amplifies the original signals and converts them to standard voltage signals which are converted into digital signals through ADC. The microcontroller doesn't only storage and preprocess digital signal but also analyzes, processes, storages, identifies and outputs signal for further through communication with upper computer.

Research Scheme of the System. The side panel crack detection system of large-scale line vibrating screen DZK2466 includes two parts of hardware circuit and software design. The hardware circuit mainly performs the acquisition and storage of data while the software can process, convert and analyze the data collected.

System Control Flow. The side panel crack signals of vibrating screen collected by sensors will be converted from analogue to digital quantum. Then, the noise reduction and fault feature extraction with wavelet must be done. Z-direction vibration signal is decomposed into three layers with wavelet packet. Then the wavelet packet energy fault feature can be obtained by calculating the signal energy of eight frequency bands after decomposition and by normalizing for them. Finally, the genetic neural network is constructed in upper computer with Matlab to diagnose the faults, so the fault type can be identified and alarmed accurately. The flow chart of the system to realize side panel cracks diagnosis of large-scale line vibrating screen DZK2466 is shown in fig.1. 


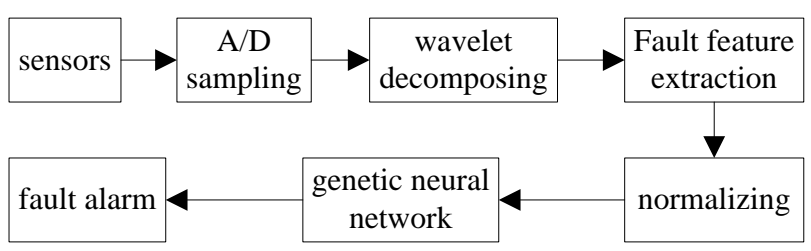

Fig.1 Flow chart of the system fault diagnosis

\section{Wavelet Analysis}

Three-axis accelerometers are placed on different position of two side panels of vibrating screen in order to acquire vibration signals with $512 \mathrm{~Hz}$ and 2048 points of sampling frequency. The basic frequency of vibrating screen DZK2466 is $16 \mathrm{~Hz}$. The normal Z-direction signal amplitude of threeaxis accelerometers at basic frequency is smaller but more high frequency interference signals exist in them. These interference signals are mainly related with environmental and operation factor through analyzing the time domain waveform and spectrum. We can conclude that the amplitude of vibration fault signal at basic frequency is bigger and the interference signals and fault frequency exist in high frequency band with test and analysis.

Data Process with Wavelet Decompositon. One-dimensional discrete wavelet transform of Zdirection vibration signal is taken and the noise reduction in every layer is done in Matlab. The soft threshold decomposition, default threshold decomposition and mandatory threshold decomposition are taken separately to reduce the noise of signals combined with concrete signal noise characteristic in Matlab programming. Wavelet db5 is selected for noise reduction by comparison. The author found that the way of setting threshold at each node respectively is better than that of setting a global threshold in reduction process. The wavelet noise reduction result with three ways of Z-direction signal is shown in fig2.
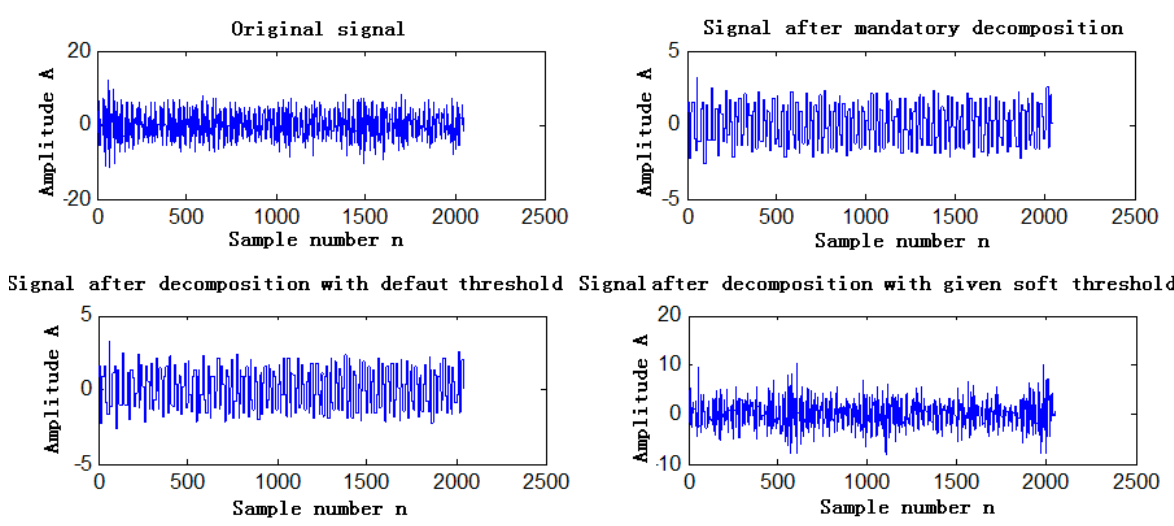

Fig.2 Processing result with three kind wavelet decomposition

By comparison we found that the relatively smooth waveform in fig. 2 is the result of mandatory decomposition with which method some useful signals may be missing. While the methods using default threshold and given soft threshold guarantee the integrity of original signal, which are more effective in the practical application. The author adopts the way of given soft threshold to reduce the noise of signal in this paper

Fault Feature Extraction of Vibrating Screen. The cracks of vibrating screen side panel are divided into four levels according to the actual production and maintenance requirements of largescale line vibrating screen DZK2466. Four kinds of crack length and the corresponding hierarchy is shown in Table 1.

Table1 Table of crack scale

\begin{tabular}{|r|r|c|r|}
\hline crack & level & crack value & level \\
\hline $0 \sim 5$ & 0 & $20 \sim 40$ & 2 \\
\hline $6 \sim 20$ & 1 & above 40 & 3 \\
\hline
\end{tabular}

Fig.3 shows different frequency waveform after being divided for three-layer with db4 wavelet of Z-direction signal of vibrating screen DZK2466 side panel. As we can see from the figure, it is high 
frequency component that creates the original spike component because there is a high agreement degree between the signal peak and the lowest frequency component while this corresponding relationship doesn't occur in other frequency components ${ }^{[4]}$.

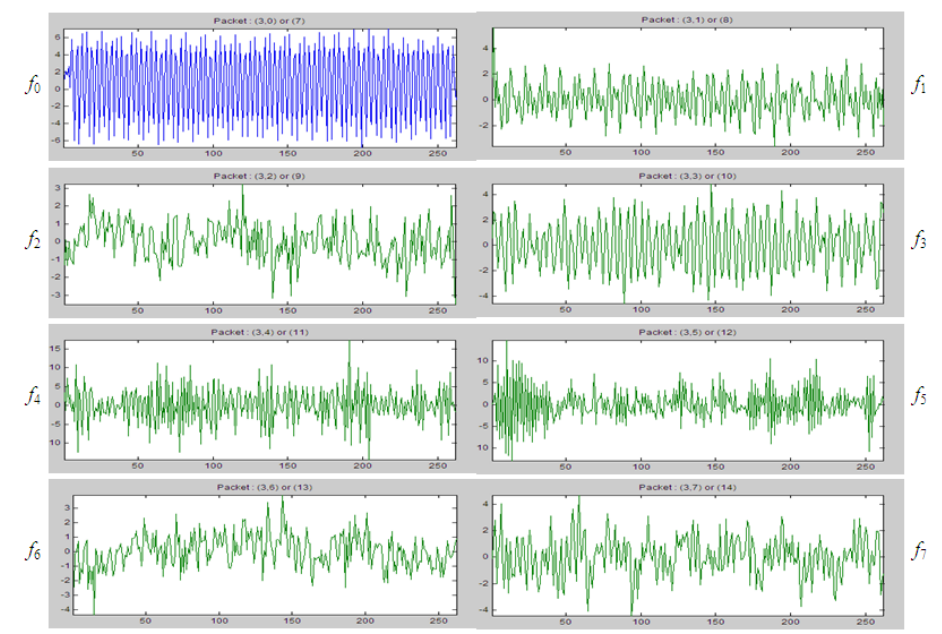

Fig.3 Signals with wavelet packet decomposition of vibrating screen side panel crack

Calculating s0 s7 for each band signal energy in Matlab, we can obtain three fault feature vector diagrams through Visual Basic programming in upper computer which are shown in Fig.4. We can see from wavelet packet energy distribution of frequency bands after normalization that the energy frequency band distribution of different state is also different obviously which can be used to represent the types of fault states. Therefore, it is an efficient fault feature extraction way.

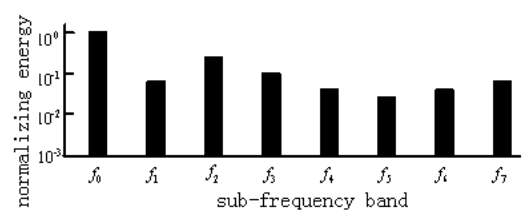

(a) fatigue crack

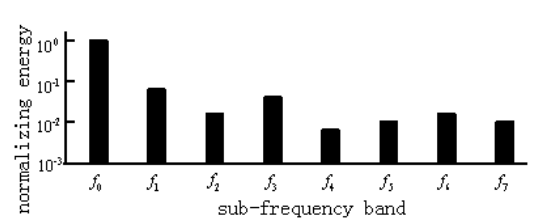

(b) crack of formation period

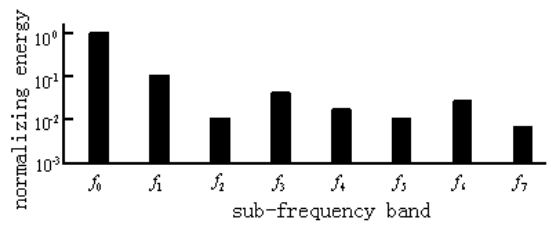

(c) shaped crack

Fig.4 Fault feature vectors of three cracks

\section{System Fault Diagnosis}

Wavelet Genetic Neural Network. Bp neural network ${ }^{[5,6]}$ of large-scale vibrating screen DZK2466 to identify side panel cracks is established targeting wavelet packet energy fault feature inputs. The neural network is optimized with genetic algorithm and is trained and simulated in Matlab ${ }^{[7,8]}$. The classification and recognition of vibrating screen side panel cracks is realized by observing input characteristic vector value and output value. The fault diagnosis accuracy of this wavelet genetic neural network can reach more than 96\% with higher fault diagnosis accuracy and speed.

Upper Computer Diagnosis System Based on Visual Basic. In upper computer software design, the real time data in single chip microprocessor is called by Visual Basic program. While function module in Matlab is called through programming interface provided by Matlab so as to realize wavelet decomposition, energy calculation and normalization ${ }^{[9]}$. The above process result can be stored into the data base in order to be called out for neural network for diagnosis. The designed visual diagnosis interface in upper computer includes main interface and every functional interfaces. Fig. 5 and fig. 6 show the wavelet analysis interface and the neural network diagnosis interface in upper computer separately.

In fig. 5 of wavelet analysis window, the acquisition signal of corresponding channel can be checked selectively. The wavelet packet decomposition program of Matlab can be directly called and the signal waveform after noise reduction can also be displayed by clicking the "Noise reduction" button. The layer number and the thresholds of every layer can be set separately through this window. These parameters can be embedded in Matlab noise reduction program automatically once setting has been finished. So we realize the intelligent soft threshold decomposition processing. 
At the same time, wavelet packet energy calculation can be finished and the fault feature can be displayed in form of bar chart by selecting automatic energy calculation. In neural network diagnosis window, the detection signals of four channels can be diagnosed separately. Fig.6 shows that the diagnosis result of channel 1 is "Early fatigue crack!".

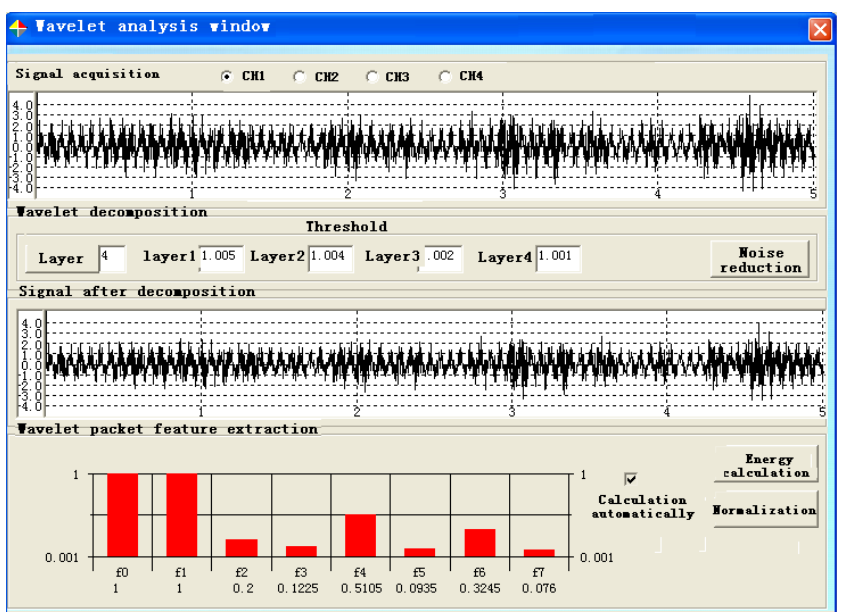

Fig.5. Wavelet analysis interface in upper computer

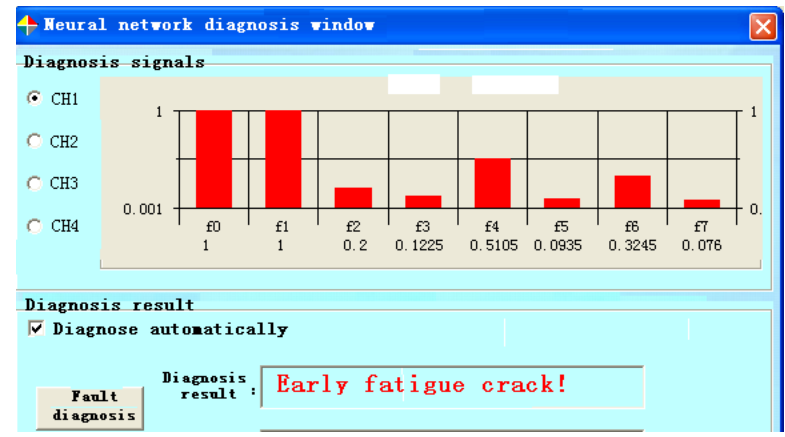

Fig.6 Neural network diagnosis interface

\section{Summary}

This paper takes side panel crack fault of large-scale line vibrating screen DZK2466 as research object and constructs the fault diagnosis system based on wavelet analysis. The function of wavelet analysis in signal noise reduction and fault feature extraction are dealt with in detail. Meanwhile, the genetic neural network in matlab is constructed through training and simulation for fault identification. Data automatic acquisition, noise reduction and fault feature extraction has been performed with upper computer diagnosis system which can be executed in real time. The practical application shows that this system has high crack identification accuracy and fault diagnosis speed.

\section{References}

[1] Ren Zi-ting, Li Hui-bin and Jin Ting et al.. Vibration Test and Fault Analysis on Shaker of Large-scale Screen[J], Coal Mine Machinery, 2008, 29 (11): 184-187.

[2] Zhong Bing-lin and Huang Ren. "Mechanical Fault Diagnosis,"[M]. Beijing: Machinery Industry Press, 1997.

[3] Peng Yu-hua. "Wavelet Transform and Engineering Application,” [M]. Being: Science Press, 1999.

[4] Liu Hua, Cai Zheng-min and Wang Yao-she et al.,Wavelet Packet Analysis and Fault Diagnosis of the Rolling Bearigns[J].Mechanical Science and Technology, 1999, 18 (2) : 301-303.

[5] Han Li-qun. “The artificial Neural Network Theory, Design and Application”[M] . Being: Chemical Industry Press, 2002.

[6] Xu Li-na. “Neural Network Control” [M].Beijing: Electronics Industry Press, 2003.

[7] Hou Z, Gupta M M.A recurrent neural network for hierarchical control of interconnected dynamic systems[J].IEEE Transactions on Neural Networks, 2007, 18(2): 466-481.

[8] Wang Jing, Li Jing-hua and Ni Ning et al.. Restricted Searching Area Hierarchical Genetic Algorithm for UAV Path Planning[J].Journal of Detection \& Control, 2011, 33 (4): 39-43.

[9] Xue Ding-yu. "'Computer Aided Design of Control System-MATLAB Language and Application[M]. Tsinghua University Press, 2006: 51-63 